\title{
New assays might facilitate the diagnosis of Creutzfeldt-Jakob disease in living patients
}

$\mathrm{D}$ efinitive diagnosis of CreutzfeldtJakob disease (CJD) relies on postmortem analyses, and detecting biomarkers of the disease in living patients often requires multiple invasive examinations. Now, two studies published recently in the New England Journal of Medicine reveal that disease-linked prion protein can be reliably detected in patients' urine or olfactory mucosa.

Gianluigi Zanusso and his colleagues at the University of Verona, Italy, demonstrated in 2003 that prions indicative of CJD could be found in the lining of the nasal vault. Unfortunately, the invasive biopsy technique they employed was associated with considerable complications, and only yielded samples with little diagnostic utility.

More than 8 years later, Zanusso came across work by Byron Caughey and others at the National Institutes of Health, MT, USA, who were developing a technique called real-time quaking-induced conversion (RT-QuIC) to amplify prions in vitro. "When Dr Zanusso heard about our new ultrasensitive assay for prions, he suggested that we return together to the idea of testing nasal specimens," says Caughey.

Zanusso and Caughey coordinated a team that obtained samples of the olfactory neuroepithelium using a minimally invasive 'nasal brushing' procedure in 31 patients with sporadic CJD and 43 controls. The investigators also obtained samples of cerebrospinal fluid, and analysed all biological materials using the RT-QuIC procedure.

Prions propagate by recruiting normal prion protein and converting it to the misfolded disease-linked form. "The RT-QuIC analysis takes advantage of this basic propagation mechanism," Caughey explains. "Samples containing prions are immersed in solutions containing normal prion protein that has been produced in E. coli and purified. The prions then induce massive fibrillization, which is easily detected."

The RT-QuIC analysis revealed diseaselinked prions in the nasal samples from 30 of the 31 patients previously diagnosed with CJD, but none of the control patients. This result compared favourably to the analysis of cerebrospinal fluid, which was positive for CJD in only 23 of 30 patients. The cerebrospinal fluid assay was also slower to elicit a fluorescent signal, and the signal was weaker overall, suggesting the less invasive nasal brushing technique might be more effective for the diagnosis of individual patients.

The detection of infected prions on a greater scale requires different tactics, however. "It is estimated that a large proportion of Europeans have been exposed to cattle prions," says Claudio Soto of the University of Texas, USA. "However, it is not known whether these people will ever develop the clinical disease or remain as silent carriers." An efficient screening program would require very easily accessible biological material.

Soto et al. collected urine samples from 82 patients with CJD and 152 control participants. When the investigators tried to analyse the samples via western blots, they were confronted with a familiar problem: even after increasing the concentration of prion protein 100-fold, overall levels were too low for disease markers to be detected.

To better their chances, the investigators employed protein misfolding cyclic amplification (PMCA), which also exploits propagation mechanics to boost prion levels. Urine samples were mixed with brain homogenate from transgenic mice bred to express human prion protein, and these suspensions were then subjected to cycles of incubation and sonication to promote prion propagation.

After the PMCA technique, diseaselinked prions were detected in 13 of 14

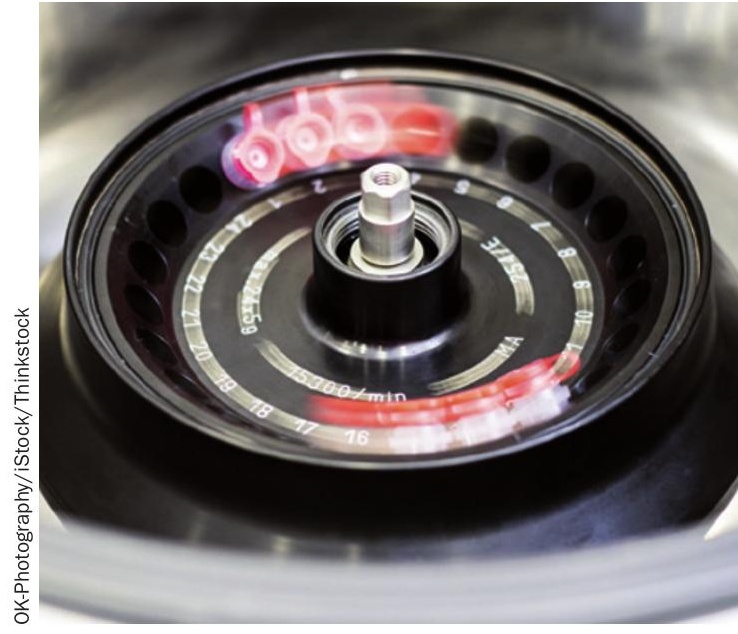

patients with variant CJD, but in none of the 68 patients with sporadic CJD or the other study participants. "Our study indicates that these two forms of CJD differ on the involvement of peripheral tissues," concludes Soto. This difference might be related to the fact that variant CJD is typically acquired by ingesting infected prions.

Both novel techniques for detecting CJD-linked prions require further validation before they can be included in medical and veternary practice. Future work must also address the possibility of iatrogenic transmission.

"The detection of substantial levels of prion seeding activity raises concerns about infectivity of CJD prions lining the nasal cavity," says Caughey. Soto is also cautious: "more than 300,000 women in the USA and Canada are prescribed urinederived gonadotropins for infertility, and it has been shown that fragments of prion protein are a frequent contaminant."

Alex Chase

Original articles Orrú, C. D. et al. A test for CreutzfeldtJakob disease using nasal brushings. N. Engl. J. Med. 371, 519-529 (2014) | Moda, F. et al. Prions in the urine of patients with variant Creutzfeldt-Jakob disease. N. Engl. J. Med. 371, 530-539 (2014) 